\title{
Quantum distillation and confinement of vacancies in a doublon sea
}

\author{
Lin Xia ${ }^{1}$, Laura A. Zundel ${ }^{1}$, Juan Carrasquilla ${ }^{1,2}$, Aaron Reinhard ${ }^{1 \dagger}$, Joshua M. Wilson ${ }^{1}$, Marcos Rigol ${ }^{1}$ \\ and David S. Weiss ${ }^{1 \star}$
}

\begin{abstract}
Ultracold atomic gases have revolutionized the study of non-equilibrium dynamics in quantum many-body systems. Many counterintuitive non-equilibrium effects have been observed, such as suppressed thermalization in a one-dimensional (1D) gas $^{1}$, the formation of repulsive self-bound dimers ${ }^{2}$, and identical behaviours for attractive and repulsive interactions ${ }^{3}$. Here, we observe the expansion of a bundle of ultracold 1D Bose gases in a flat-bottomed optical lattice potential. By combining in situ measurements with photoassociation ${ }^{4,5}$, we follow the spatial dynamics of singly, doubly and triply occupied lattice sites. The system sheds interaction energy by dissolving some doublons and triplons. Some singlons quantum distil out of the doublon centre $^{6,7}$, whereas others remain confined 7 . Our Gutzwiller mean-field model captures these experimental features in a physically clear way. These experiments might be used to study thermalization in systems with particle losses ${ }^{8}$, the evolution of quantum entanglement ${ }^{9,10}$ or, if applied to fermions, to prepare very low entropy states ${ }^{6}$.
\end{abstract}

Quantum distillation is a previously unobserved phenomenon in which atoms at singly occupied lattice sites (singlons) escape the central region of an untrapped lattice gas, leaving doubly occupied lattice sites (doublons) behind (Fig. 1a) ${ }^{6}$. It depends on one readily achievable condition, that there be an energy mismatch that prevents isolated doublons from disintegrating into two singlons. Singlons in a sea of doublons can be understood as vacancies. The tunnelling rate of a singlon in an empty lattice is $J$ and the bosonic vacancy tunnelling rate is $2 J$, because the vacancy moves when either of the adjacent doublon's atoms tunnels. When bosonic vacancies reach a doublon sea edge, only those with intermediate energies can transmit into the empty lattice while conserving energy $^{7}$, as illustrated in Fig. 1b. Vacancies with quasimomenta outside that limited range reflect from the edge, confining them in the doublon sea. When a singlon does exit, it purifies and shrinks the doublon sea by one lattice site. It has been suggested that collisions of vacancies with triplons can thermalize the vacancies, possibly transferring them into transmissible quasimomentum states ${ }^{7}$. In contrast, fermionic vacancies have the same tunnelling energy as singlons in the empty lattice, so they always pass through the edge of the doublon sea.

A previous non-equilibrium experiment with bosons in flat 1D lattices mostly focused on initial single-atom number states in a deep lattice, and studied the expansion dynamics after a quench of the on-site interaction energy ${ }^{3}$. Such a quench leaves the manybody wavefunction out of local equilibrium. In contrast, we start our experiments with trapped, superfluid, 1D Bose gases in lattices with an average of between one and two atoms per site, and we quench by suddenly removing the trap. This is a fundamentally different quench, a geometric quench, after which the many-body wavefunction is still locally in equilibrium. Geometric quenches have been theoretically shown to lead to remarkable universal phenomena, such as quasicondensation at finite momenta ${ }^{11,12}$, dynamical fermionization of the Tonks-Girardeau gas ${ }^{13,14}$, and identical expansions of bosons and fermions ${ }^{15}$. Our experimentally observed expansion dynamics are qualitatively reproduced by a Gutzwiller mean-field calculation (see Methods). By theoretically and experimentally studying the spatial evolution of site occupancy, we obtain a straightforward physical interpretation of the dynamics. It exhibits clear signatures of quantum distillation ${ }^{6}$ and confinement ${ }^{7,16}$ of vacancies in the doublon sea.

In our experiment, Bose-condensed ${ }^{87} \mathrm{Rb}$ atoms in a crossed dipole trap are slowly loaded into an array of $1 \mathrm{D}$ tubes formed by a blue-detuned 2D optical lattice (wavevector $k=2 \pi / 773 \mathrm{~nm}$ ), with a superposed axial optical lattice of variable depth $V_{0}$ and a reddetuned crossed dipole trap for overall confinement (see Methods). For the density and $V_{0}$ used in this work, the initial ground states are predominantly superfluid ${ }^{17}$. As each lattice site starts with a superposition of number states, pictures such as those in Fig. 1a represent one of many distributions whose coherent sum is the state of the system. As we will see, most of the qualitative behaviour of what are thus delocalized singlons and doublons can be understood using localized pictures, leavened by the understanding that each picture represents only a small piece of the overall wavefunction.

At $t_{\mathrm{ev}}=0$, we suddenly lower the depth of the crossed dipole trap, leaving enough power to cancel the residual anti-trap due to the $2 \mathrm{D}$ lattice beams over a range of $\sim 160 \mu \mathrm{m}$ (see Methods). We observe the subsequent spatial evolution in three ways (referred to as M1, M2 and M3), which together allow us to separately determine the spatial evolution of the probability distributions of singlons, doublons and atoms at more highly occupied sites. In M1, we measure all the atoms after a given time $t_{\mathrm{ev}}$ by switching to a $1 \mathrm{D}$ axial lattice of depth $27 E_{\text {rec }}$ (where the recoil energy $E_{\text {rec }}=\hbar^{2} k^{2} / 2 M$ and $M$ is the $\mathrm{Rb}$ mass) and allowing the atoms to expand radially so that the density is low enough for absorption imaging. Information about the transverse distribution among tubes is lost, but the axial distribution is preserved, with a resolution of $\sim 3 \mu \mathrm{m}$. In M2, at $t_{\mathrm{ev}}$ we suddenly switch to a $27 E_{\text {rec }}$ lattice in each of three directions and turn on a photoassociation pulse for $1.5 \mathrm{~ms}$ (refs 4,5 ), which is long enough to eliminate all doublons, $2 / 3$ of the triplon atoms, and most of the atoms from sites with higher occupancies. The axial distribution measurement is then made as in M1. In M3, at $t_{\mathrm{ev}}$ we suddenly switch to the $27 E_{\text {rec }} 3 \mathrm{D}$ lattice and wait for $50 \mathrm{~ms}$, which is long enough for

\footnotetext{
${ }^{1}$ Department of Physics, The Pennsylvania State University, University Park, Pennsylvania 16802, USA. ${ }^{2}$ Perimeter Institute for Theoretical Physics, Waterloo, Ontario N2L 2Y5, Canada. †Present address: Department of Physics, Otterbein University, Westerville, Ohio 43081, USA.

*e-mail: dsweiss@phys.psu.edu
} 
a

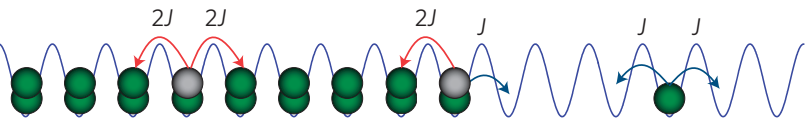

b

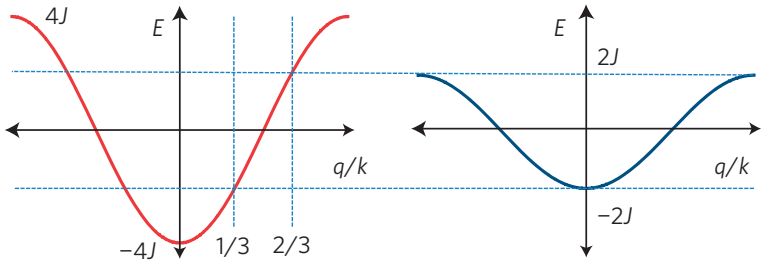

Figure 1 | Boson quantum distillation. $\mathbf{a}, \mathrm{A}$ singlon among doublons acts as a vacancy (shown in grey), which tunnels through the doublon sea at $2 \mathrm{~J}$. A singlon in the empty lattice tunnels at $J$. A vacancy at the edge of the doublon sea is the same as a singlon at the edge of the empty lattice. It is important to note that, at our low temperature and our lattice depths, atoms are delocalized. Each 1D tube is occupied by a superposition of many distributions like the one pictured, generally including sites with higher occupancies and empty sites, as well as different doublon edge positions. b. Ground energy bands within the doublon sea (on the left) and in the empty lattice (on the right). Only atoms with quasimomentum between $1 / 3$ and $2 / 3$ of the band edge can tunnel from the doublon sea into the empty lattice. Outside that range, transmission into the empty lattice does not conserve energy. This limitation on quantum distillation is absent for fermions.

three-body inelastic collisions ${ }^{18,19}$ to empty the triplon sites and most atoms from more highly occupied sites. The axial distribution is then measured as in M1.
Figure $2 \mathrm{a}-\mathrm{c}$ shows the evolution of the total atom distribution (M1) for $V_{0}=3 E_{\text {rec }}, 4 E_{\text {rec }}$ and $5 E_{\text {rec }}$. These depths correspond to $U / J$ of 4.7, 6.8 and 9.6 (ref. 20), respectively, in the one-band Hubbard model ${ }^{21,22}$, where $U$ is the on-site repulsion energy. All are characterized by a central core of atoms that steadily releases atoms that tunnel away from the centre. Figure $2 \mathrm{~d}-\mathrm{f}$ shows the distributions of single atoms, which are derived from M1, M2 and M3 (see Supplementary Fig. 1a-f). These curves show two dominant features. First, the cores contain many single atoms. Second, the broader pedestals of the M1 distributions are composed nearly exclusively of single-atom sites. The velocities of the leading edges of the pedestals equal, to within $\sim 10 \%$ systematic uncertainties, the calculated maximum possible velocity $\left(v_{\max }=2 \mathrm{Ja} / \hbar\right.$, where $a=\lambda / 2$ is the lattice spacing) for single atoms tunnelling in the lowest band (see insets in Fig. $2 \mathrm{a}-\mathrm{c}$ ). These velocities start to decrease at the end of the compensation range of the crossed dipole trap, ultimately Bragg scattering backwards; we do not show data after atoms return to the core. Figure $2 \mathrm{~g}-\mathrm{i}$ shows the distribution of doublons, derived from all three measurement types. The number of doublons decreases steadily after the quench, but the widths of the doublon distributions barely change. The triplon distributions (see Supplementary Fig. 1g-i) have the same width as the doublon distributions to within a $10 \%$ uncertainty.

Figure $2 \mathrm{j}-\mathrm{l}$ (and Supplementary Fig. $1 \mathrm{j}-\mathrm{l}$ ) shows the results of a Gutzwiller mean-field calculation ${ }^{16,23}$ (see Methods), which simulates an array of identical tubes with different atom numbers, as in the experiment, and discretizes the direction along the tubes ${ }^{24}$ so that the standard single-band approximation is not used. The theory assumes an initial zero temperature Bose-Einstein condensate. This means that finite temperature and quantum fluctuations due to the $1 \mathrm{D}$ character of the system are not taken into account. The initial

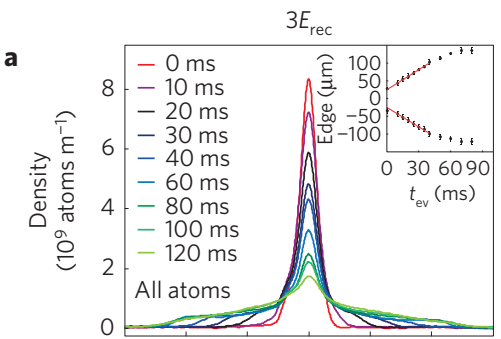

d

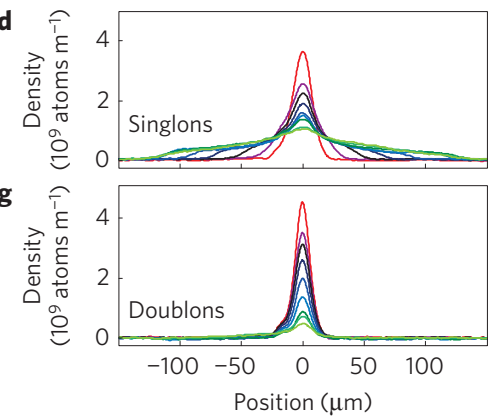

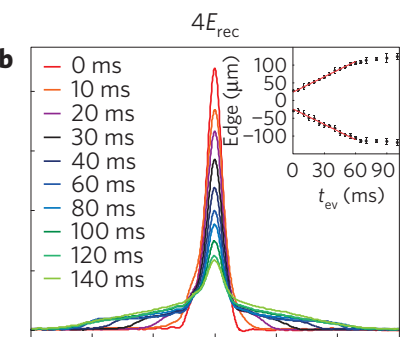
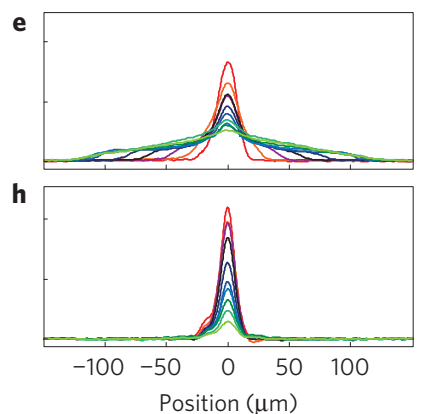
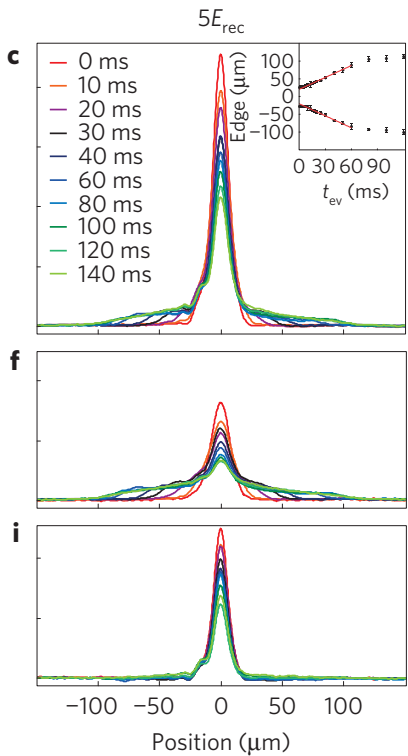
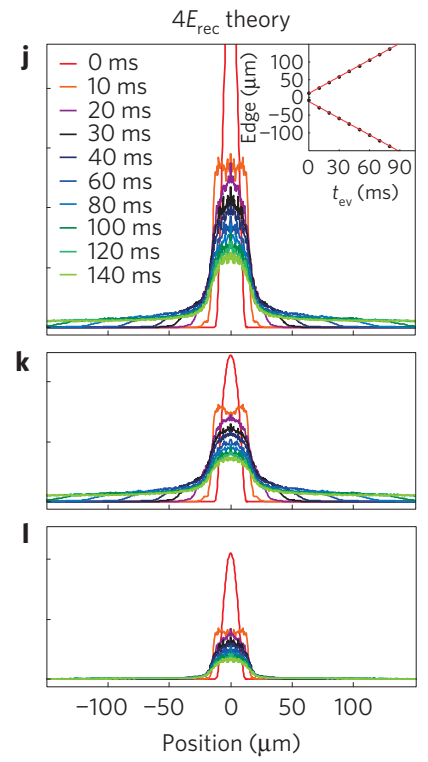

Figure 2 | Spatial dynamics in the flat-bottomed lattice. a-c, M1 measurements of the total atom distributions at successive $t_{\mathrm{ev}}$ at $V_{0}=3 E_{\mathrm{rec}}, 4 E_{\mathrm{rec}}$ and $5 E_{\text {rec }}$, respectively. Insets: locations of the distribution edges versus evolution time. The measured velocity values for $V_{0}=3 E_{\text {rec }}, 4 E_{\text {rec }}$ and $5 E_{\text {rec }}$ are $1.9 \pm 0.18,1.44 \pm 0.02$ and $1.12 \pm 0.03 \mathrm{~mm} \mathrm{~s}^{-1}$, respectively, slightly slower than $v_{\max }$ of $2.1,1.6$ and $1.2 \mathrm{~mm} \mathrm{~s}^{-1}$. $\mathbf{d}-\mathbf{f}$, Distributions of singlons at successive $t_{\mathrm{ev}}$ at $V_{0}=3 E_{\mathrm{rec}}, 4 E_{\mathrm{rec}}$ and $5 E_{\mathrm{rec}}$, respectively. These are derived by combining the raw measurements according to the formula $M 2-(M 1-M 3) / 3$. $\mathbf{g}-\mathbf{i}$, Distributions of doublons at successive $t_{\mathrm{ev}}$ at $V_{0}=3 E_{\mathrm{rec}}, 4 E_{\mathrm{rec}}$ and $5 E_{\mathrm{rec}}$, respectively. These are derived by combining the raw measurements according to the formula $M 1-M 2-2(M 1-M 3) / 3$. Note that all the experimental plots $(\mathbf{a}-\mathbf{i})$ have the same vertical scale. For a discussion of the small asymmetry in these figures, see Methods. $\mathbf{j}-\mathbf{I}$, Results according to Gutzwiller mean-field theory for $V_{0}=4 E_{\text {rec }}$ for the distribution of all atoms, singlons and doublons, respectively. We choose the theoretical initial conditions so that the fraction of singlons matches the experiment (see Methods). The initial fraction of triplons and higher exceed what is seen in the experiment, and the initial cloud length is smaller. The qualitative behaviour seen in the theory (and in the experiment) is robust to the initial conditions. All but the least occupied $\sim 20 \%$ of tubes behave qualitatively like the average over all tubes, but with less doublon dissolution and quantum distillation in the most-occupied tubes. The inset of $\mathbf{j}$ shows the motion of the edges at $v_{\max }$ as expected when the single-particle quasimomentum distribution includes the midpoint of the band. 

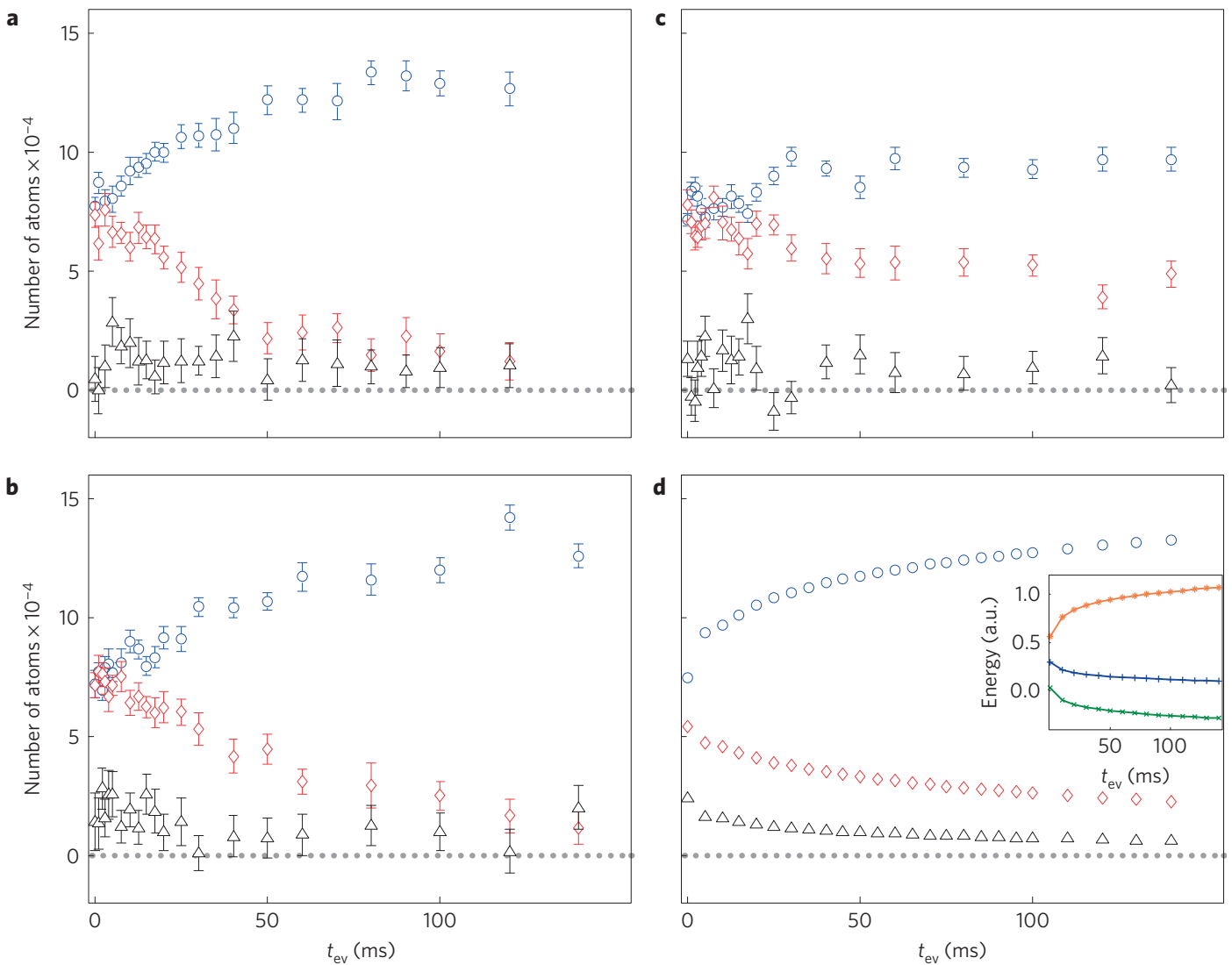

Figure 3 | Time evolution of singlons, doublons and triplons. a-c, Experimental results for $V_{0}=3 E_{\text {rec }}(\mathbf{a}), V_{0}=4 E_{\text {rec }}(\mathbf{b})$ and $V_{0}=5 E_{\text {rec }}(\mathbf{c})$. The error bars derive from the standard deviations determined from eight measurements. When the curves in $\mathbf{a}-\mathbf{c}$ are plotted together with a time axis rescaled by $J$ (see Supplementary Fig. 2), they overlap well until the doublons reach their asymptotic number. $\mathbf{d}$, Theoretical results for $V_{0}=4 E_{\text {rec. }}$. In all plots singlons are shown as blue circles, doublons as red diamonds and triplons as black triangles. Inset in $\mathbf{d}$ shows the interaction energy (blue pluses), kinetic energy (orange asterisks) and lattice potential energy (green crosses) versus $t_{\mathrm{ev}}$ calculated for $V_{0}=4 E_{\text {rec }}$.

distribution is thus an imperfect match to the experiment (see Methods). The doublons initially expand farther in the theory than in the experiment, presumably because of the long-range initial phase coherence in the theory. The early decrease in the theory's doublon density no doubt affects the details of the ensuing dynamics; however, qualitatively, the theory behaves like the experiment in all respects other than the shape and size of the doublon distributions. For further comparison to theory, see Supplementary Information.

Figure $3 \mathrm{a}-\mathrm{c}$ shows the number of singlons, doublons and triplons as a function of time, derived from the appropriate combinations of M1, M2 and M3. The numbers of doublons and triplons drop steadily, with corresponding increases in the numbers of singlons. The theory shows similar behaviour (see Fig. 3d). Although conservation of energy dictates that isolated doublons cannot dissociate for $U / J \gtrsim 4$ (ref. 2 ), in a predominantly doublon sea the aforementioned tunnelling enhancement doubles this limit. Similarly, one can show that a sea of singlons increases the limit by $50 \%$. That our doublons live in a bath intermediate to these two seas explains why they dissociate, at least for $U / J \lesssim 8$, and why the dissociation rate decreases at long times (see especially Fig. 3a after $50 \mathrm{~ms}$ ) when the number of empty sites in the centre increases. Dissociation for $U / J=9.6\left(V_{0}=5 E_{\text {rec }}\right)$ naively requires that energy be shared among more singlons ${ }^{25,26}$, but it might be that the one-band Hubbard model calculation $^{20}$ overestimates the effective value of $U / J$. The latter interpretation is supported by the fact that, at short times, the evolutions of all the curves in Figs 2 and 3 are approximately self-similar when the time axes are multiplied by $J$ (see Supplementary Fig. 2), to within the small differences in the doublon distribution widths discussed below. Although the physics is dominated by interacting particle effects, marginal changes in site occupancy scale with $J$. Our mean-field calculation allows us to explicitly track the conversion of potential energy (interaction + lattice potential) into kinetic energy (see inset) that results primarily from doublon dissolution.

Quantum distillation is difficult to isolate at early times, because it occurs while initially unconfined singlons are also leaving the central region and singlons are being created by dissolution. But quantum distillation dominates at $5 E_{\text {rec }}$ (Fig. 2f) after $20 \mathrm{~ms}$, by which time the doublon number is stable (see also Figs $2 \mathrm{i}$ and $3 \mathrm{c}$ ) and the unconfined singlons present a locally flat background. The number of singlons confined in the doublon sea as a function of time is plotted in Fig. 4a (see Methods). Its steady decrease is a clear signature of quantum distillation, further supported by the fact that the rate scales with $J$ (see also Supplementary Information). At late times, the fraction of confined singlons levels off at $~ 5 \%$, showing long-term vacancy confinement in the doublon sea. The mean-field calculations also show some singlons initially leaving and others remaining indefinitely, but fewer singlons distil out in the calculations. This is expected because the real $1 \mathrm{D}$ gas has a broader initial quasimomentum distribution. Thus, in the calculation there are more singlons with the lower energies that do not transmit out of the doublon sea.

In $J$-rescaled time, after doublons stop dissolving at $5 E_{\text {rec }}$ they are still dissolving at lower lattice depths. That the three sets of data points in Fig. 4a overlap means that extra doublon dissolution does not affect the central singlon number. This could be because the vast majority of singlons created when doublons dissolve have the right quasimomentum for immediate quantum distillation, and leave the centre rapidly. 


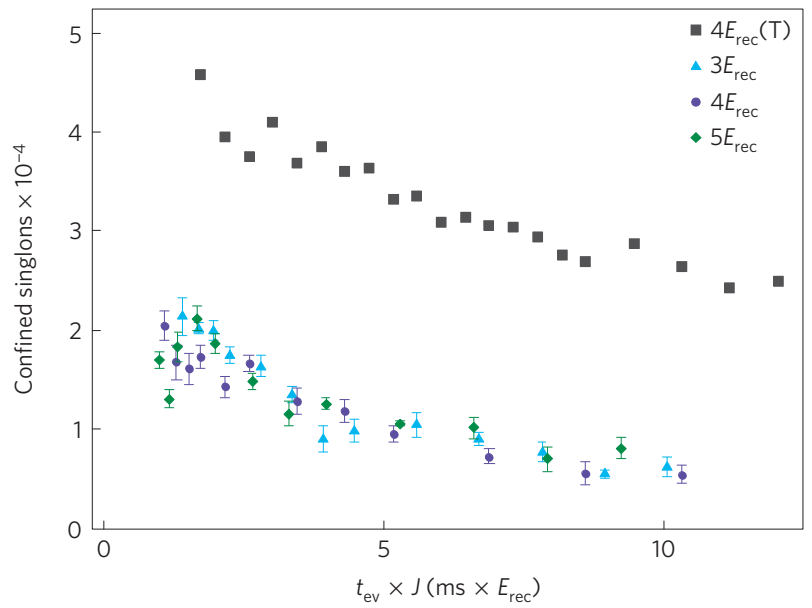

b

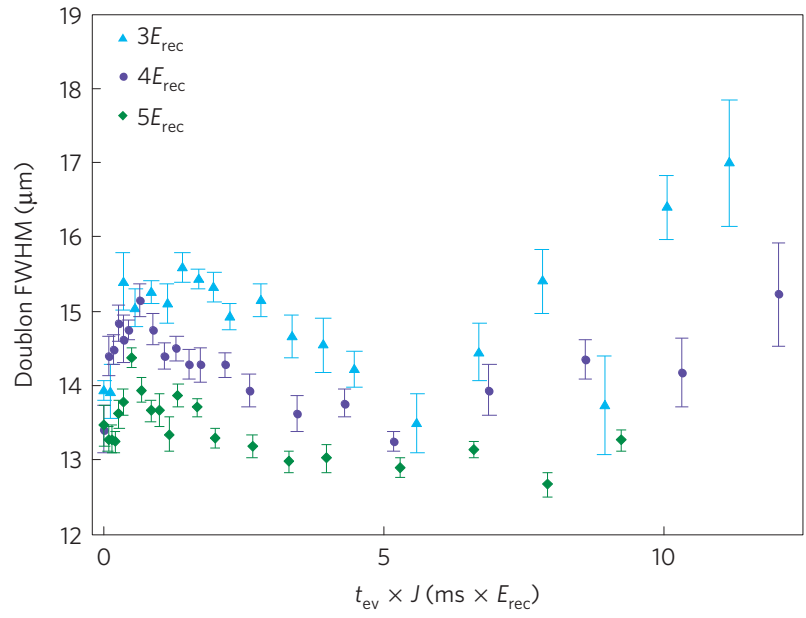

Figure 4 | Tunnelling-rescaled dynamics. a, Number of singlons confined in the doublon sea versus the tunnelling-rescaled evolution time. The triangles, circles and diamonds represent $V_{0}=3 E_{\text {rec }}, 4 E_{\text {rec }}$ and $5 E_{\text {rec }}$, respectively, in the experiment. The squares represent the theoretical results for $V_{0}=4 E_{\text {rec }}$. See Methods for a description of how the data is derived from the singlon distributions. b. FWHM of the doublon distributions versus the tunnelling-rescaled evolution time (same labels as in a). The error bars in this figure derived from the standard deviation of eight measurements.

Further evidence of quantum distillation is given in Fig. 4b, which shows the evolution of the full width at half maximum (FWHM) of the doublon distribution. There are three size-changing processes, each dominating for a time. The FWHMs increase during the first $10-20 \mathrm{~ms}$ because doublons can initially expand into singly occupied sites and perhaps there is more doublon dissolution in the middle (see Fig. 2g-i). The FWHMs then decrease owing to the mechanics of distillation, where escaping singlons move the last doublon one site inward. When the rate of quantum distillation decreases (near $4 \mathrm{~ms} \cdot E_{\text {rec }}$ in Fig. $4 \mathrm{a}$ ) then, as long as the lattice depth is small enough, shrinking is overtaken by expansion. We suspect this to be the result of higher-order processes involving confined singlons that compromise the stability of the edges of the doublon sea ${ }^{27}$. This unanticipated higher-order effect, undoubtedly absent in fermions and not present in the mean-field theory results, further limits the effectiveness of bosonic quantum distillation in producing low-entropy blocks of doublons. The approximate stability or slight increase in the width of the doublon distribution implies that, as time evolves, the number of empty sites among the doublons increases.

Our work has concentrated on spatial distributions, which are local properties, but it should also be possible to use related techniques such as time-of-flight measurements to study non-local properties, such as quasimomentum distributions and correlations. This simple lattice system, in which the doublon sea is open but nonetheless settles to a stable steady state, can help address major open questions in quantum dynamics, such as how systems thermalize in the presence of particle losses ${ }^{8}$. Studying how quantum correlations grow after the quench should give a more general insight into how entanglement spreads in quantum systems ${ }^{9}$. The fact that our mean-field treatment (exact only in infinite dimensions) qualitatively captures the 1D dynamics, suggests that similar dynamics occur in higher dimensions. Finally, an experimental implementation with fermions holds the promise of producing doublon cores with superlatively low entropy ${ }^{6}$, which might allow the study of hitherto inaccessible models of quantum magnetism ${ }^{28}$ and high-temperature superconductivity ${ }^{29}$.

\section{Methods}

Experiment. Trapping: We start with $2 \times 10^{5}$ Bose-condensed ${ }^{87} \mathrm{Rb}$ atoms in the $F=1, m_{F}=1$ state in a crossed dipole trap ${ }^{30}$ made from $1.064 \mu \mathrm{m}$ laser beams, with $1.8 \mathrm{~W}$ per beam and $160 \mu \mathrm{m}$ beam waists. Gravity is cancelled by a magnetic field gradient. A blue-detuned $773.5 \mathrm{~nm}$ wavelength $3 \mathrm{D}$ optical lattice, made from two retroreflected horizontal $450 \mu \mathrm{m}$ waist beams and a retroreflected vertical $700 \mu \mathrm{m}$ waist beam, is turned on in $14 \mathrm{~ms}$ to a depth of $V_{0}$, after which the two horizontal lattice beam pairs are increased to their full depth of $40 E_{\text {rec }}$ in $44 \mathrm{~ms}$. We find that the results of the experiment (including spatial distributions and occupancy fractions) do not change significantly as long as the lattice turn-on times are $35 \mathrm{~ms}$ or longer. That remains true if we wait for tens of $\mathrm{ms}$ in all the traps before starting the evolution.

Flat lattice: As for technical reasons the lattice waists are much larger than the dipole trap waists, we can create a flat lattice only near the centre of the trap. To fine-tune the cancellation of the two potentials, we start with trapped $1 \mathrm{D}$ gases with no axial lattice, and choose the highest crossed dipole beam intensity at which no atoms remain trapped in the central region. This gives a central potential that is flat to within $0.08 E_{\text {rec }}$ over a length of $160 \mu \mathrm{m}$.

Small bump: The small bump on the left of the initial atom distributions in the experimental results of Fig. 2 is due to spatial imperfections on the beams that make up the confining crossed dipole trap, where the atoms are trapped 3.4 Rayleigh lengths from the beam focus. After $t_{\mathrm{ev}}=0$ the atoms evolve in a much smoother potential, so only the initial distribution is affected by this issue, not the evolution. The bump can serve as a feature, because it provides confirmatory evidence of the fraction of singlons that remain confined (see, for example, the long-time curves in Fig. 2f).

Determination of the trapped singlon fraction: We analyse the singlon distributions (see Fig. 2d-f), by first determining the FWHM of the doublons (see Figs $2 \mathrm{~g}-\mathrm{i}$ and $4 \mathrm{~b}$ ) at each time. We then measure the difference between the peak of the singlon distribution and its value at the doublon FWHM positions. We assume that the confined peak height is twice that difference, and has the doublon width. We do not use this procedure at very early times, before there is a discernible shoulder in the singlon distribution, although the curves in Fig. $4 \mathrm{a}$ change little if we add a few earlier points. The assumption that the FWHMs of doublons and trapped singlons are the same is not exactly true for a bundle of tubes. This procedure also assumes that the unconfined singlon distribution is approximately flat in the central region, which is also not exactly true. Our confidence in the reliability of this procedure is buttressed by the universality of the curves in Fig. 4a, which holds despite the systematic differences in the doublon FWHMs at different $V_{0}$ (Fig. $4 \mathrm{~b}$ ).

Theoretical calculations. For the theoretical analysis of the expansion, we model a collection of independent 1D tubes. To account for possible higher-band effects during the dynamics, which may be important for the lowest lattice depths, we do not use the one-band approximation that results in the standard Bose-Hubbard model. Instead, we discretize the space along the tubes by introducing an artificial grid of spacing $\ell \ll \lambda$ (we take $\ell=0.05 \lambda$, where $\lambda$ is the wavelength of the optical lattice) to obtain a representation of the continuum in terms of an artificial 1D Bose-Hubbard model ${ }^{24}$. The particles are then subject to the periodic potential generated by the optical lattice and to the confining potentials generated by the crossed optical dipole trap. We simulate arrays of up to $110 \times 110$ tubes, with each tube's length being $500 \lambda$. We choose the theoretical initial conditions so that the fraction of singlons in the simulation matches the experiment. This is done by arbitrarily tuning the strength of the $t_{\mathrm{ev}}<0$ axial and transverse trapping potentials, keeping their ratio fixed to the experimental value. The parameters for $t_{\mathrm{ev}} \geq 0$ are those in the experimental set-up, except that the overall confining potential is set exactly to zero. The calculations are carried out within the Gutzwiller mean-field approximation ${ }^{16,23}$, where the initial state is selected to be 
the ground state in the absence of tunnelling between the 1D tubes. Our results are robust to further reduction of the value of $\ell$. For further details, see Supplementary Information.

Received 27 October 2014; accepted 8 January 2015; published online 16 February 2015

\section{References}

1. Kinoshita, T., Wenger, T. \& Weiss, D. S. A quantum Newton's cradle. Nature 440, 900-903 (2006).

2. Winkler, K. et al. Repulsively bound atom pairs in an optical lattice. Nature 441, 853-856 (2006).

3. Ronzheimer, J. P. et al. Expansion dynamics of interacting bosons in homogeneous lattices in one and two dimensions. Phys. Rev. Lett. 110, 205301 (2013).

4. Theis, M. et al. Tuning the scattering length with an optically induced Feshbach resonance. Phys. Rev. Lett. 93, 123001 (2004).

5. Kinoshita, T., Wenger, T. \& Weiss, D. S. Local pair correlations in one-dimensional Bose gases. Phys. Rev. Lett. 95, 190406 (2005).

6. Heidrich-Meisner, F. et al. Quantum distillation: Dynamical generation of low-entropy states of strongly correlated fermions in an optical lattice. Phys. Rev. A 80, 041603(R) (2009).

7. Muth, D., Petrosyan, D. \& Fleischhauer, M. Dynamics and evaporation of defects in Mott-insulating clusters of boson pairs. Phys. Rev. A 85, 013615 (2012).

8. Makotyn, P., Klauss, C. E., Goldberger, D. L., Cornell, E. A. \& Jin, D. S. Universal dynamics of a degenerate unitary Bose gas. Nature Phys. 10, 116-119 (2014).

9. Cheneau, M. et al. Light-cone-like spreading of correlations in a quantum many-body system. Nature 481, 484-487 (2012)

10. Daley, A. J., Pichler, H., Schachenmayer, J. \& Zoller, P. Measuring entanglement growth in quench dynamics of bosons in an optical lattice. Phys. Rev. Lett. 109, 020505 (2012).

11. Rigol, M. \& Muramatsu, A. Emergence of quasicondensates of hard-core bosons at finite momentum. Phys. Rev. Lett. 93, 230404 (2004).

12. Rodriguez, K., Manmana, S. R., Rigol, M., Noack, R. M. \& Muramatsu, A Coherent matter waves emerging from Mott-insulators. New J. Phys. 8 , 169 (2006).

13. Rigol, M. \& Muramatsu, A. Fermionization in an expanding 1D gas of hard-core bosons. Phys. Rev. Lett. 94, 240403 (2005)

14. Minguzzi, A. \& Gangardt, D. M. Exact coherent states of a harmonically confined Tonks-Girardeau gas. Phys. Rev. Lett. 94, 240404 (2005).

15. Vidmar, L. et al. Sudden expansion of Mott insulators in one dimension. Phys Rev. B 88, 235117 (2013).

16. Jreissaty, A., Carrasquilla, J. \& Rigol, M. Self-trapping in the two-dimensional Bose-Hubbard model. Phys. Rev. A 88, 031606 (2013).

17. Rigol, M., Batrouni, G. G., Rousseau, V. G. \& Scalettar, R. T. State diagrams for harmonically trapped bosons in optical lattices. Phys. Rev. A 79, 053605 (2009).
18. Burt, E. A et al. Coherence, correlations, and collisions: What one learns about Bose-Einstein condensates from their decay. Phys. Rev. Lett. 79, 337-340 (1997).

19. Tolra, B. L. et al. Observation of reduced three-body recombination in a correlated 1D degenerate Bose gas. Phys. Rev. Lett. 92, 190401 (2004).

20. Walters, R., Cotugno, G., Johnson, T. H., Clark, S. R. \& Jaksch, D. Ab initio derivation of Hubbard models for cold atoms in optical lattices. Phys. Rev. A 87, 043613 (2013)

21. Fisher, M. P. A., Weichman, P. B., Grinstein, G. \& Fisher, D. S. Boson localization and the superfluid-insulator transition. Phys. Rev. B 40, 546-570 (1989).

22. Cazalilla, M. A., Citro, R., Giamarchi, T., Orignac, E. \& Rigol, M. One dimensional bosons: From condensed matter systems to ultracold gases. Rev. Mod. Phys. 83, 1405-1466 (2011).

23. Jaksch, D., Venturi, V., Cirac, J. I., Williams, C. J. \& Zoller, P. Creation of a molecular condensate by dynamically melting a Mott insulator. Phys. Rev. Lett 89, 040402 (2002).

24. Stoudenmire, E. M., Wagner, L. O., White, S. R. \& Burke, K. One-dimensional continuum electronic structure with the density-matrix renormalization group and its implications for density-functional theory. Phys. Rev. Lett. 109, 056402 (2012)

25. Strohmaier, N. et al. Observation of elastic doublon decay in the Fermi-Hubbard model. Phys. Rev. Lett. 104, 080401 (2010).

26. Sensarma, R. et al. Lifetime of double occupancies in the Fermi-Hubbard model. Phys. Rev. B 82, 224302 (2010).

27. Petrosyan, D., Schmidt, B., Anglin, J. R. \& Fleischhauer, M. Quantum liquid of repulsively bound pairs of particles in a lattice. Phys. Rev. A 76, 033606 (2007).

28. Balents, L. Spin liquids in frustrated magnets. Nature 464, 199-208 (2010).

29. Esslinger, T. Fermi-Hubbard physics with atoms in an optical lattice. Ann. Rev. Condens. Matter Phys. 1, 129-152 (2010).

30. Kinoshita, T., Wenger, T. \& Weiss, D. S. All-optical Bose-Einstein condensation using a compressible crossed dipole trap. Phys. Rev. A 71, 011602 (2005).

\section{Acknowledgements}

We are indebted to A. Daley, M. Fleischauer and F. Essler for illuminating conversations. This work was supported by NSF Grant No. PHYS-167830, the Army Research Office, and the Office of Naval Research (J.C. and M.R.). J.C. acknowledges support from the John Templeton Foundation. Research at Perimeter Institute is supported through Industry Canada and by the Province of Ontario through the Ministry of Research and Innovation.

\section{Author contributions}

All authors contributed significantly to this work.

\section{Additional information}

Supplementary information is available in the online version of the paper. Reprints and permissions information is available online at www.nature.com/reprints. Correspondence and requests for materials should be addressed to D.S.W.

\section{Competing financial interests}

The authors declare no competing financial interests. 